\title{
Evaluation of Ofloxacin and Ornidazole as an Adjunct to Scaling and Root Planing in the Treatment of Generalized Chronic Periodontitis: A Randomized Clinical Study
}

\author{
Athira K Chermmal ${ }^{1}$, Harsha M Babu ${ }^{2}$, Marenahalli R Vivekananda ${ }^{3}$
}

\begin{abstract}
Aim and objective: Over the years, treatment for chronic periodontitis is changing owing to an improved understanding of the pathogenesis of the periodontal disease. Tissue invasive properties of periodontal pathogens have necessitated the use of antimicrobial agents as an adjunct to mechanical plaque control in the treatment of periodontitis. Hence, the study aimed to evaluate the efficacy of ofloxacin-ornidazole combination therapy as an adjunct to scaling and root planing in the treatment of generalized chronic periodontitis.

Materials and methods: A total of 40 study participants, aged from 25 to 55 years, with generalized chronic periodontitis were selected. They were randomly divided into two groups-Group I: receiving scaling and root planing along with ofloxacin-ornidazole combination therapy and group II: receiving scaling and root planing. Gingival index (GI), sulcular bleeding index (SBI), and probing pocket depth (PPD) scores were recorded at baseline, 14th day, and after 1 month.

Results: Both the groups showed a reduction in GI scores, SBI scores, and mean pocket probing depth from baseline to 14 th day and 1 month. However, the improvement in scores was better in a group with the ofloxacin-ornidazole combination, than the group with scaling and root planing alone. The intergroup comparison of the gingival indices, SBI, and mean pocket probing depth at baseline, 14 th day, and 1 month have shown the statistically significant difference.

Conclusion: It can be concluded that ofloxacin-ornidazole combination therapy is well tolerated by the patients, safe, favorable patient compliance, and proved to be effective in patients with generalized chronic periodontitis when used as an adjunct to mechanical plaque control. Clinical significance: Ofloxacin-ornidazole combination can be used as an adjuvant in the treatment of generalized chronic periodontitis. Keywords: Chronic periodontitis, Ofloxacin, Ornidazole, Root planing, Scaling.

World Journal of Dentistry (2021): 10.5005/jp-journals-10015-1881
\end{abstract}

\section{INTRODUCTION}

Chronic periodontitis is the most common infectious disease leading to the destruction of tooth-supporting tissues, bacterial plaque being the major etiological factor. Among the wide range of species found in the dental plaque, specific subgingival microbial agents are found to cause destructive periodontitis. ${ }^{1}$ With the advancement of the disease, bacterial biofilm extends far subgingivally that the patient cannot reach it during oral hygiene maintenance. ${ }^{2}$ Periodontal pathogens such as Porphyromonas gingivalis and Aggregatibacter actinomycetemcomitans possess the capability of invading the periodontal pocket epithelium, making it difficult to eliminate by conventional non-surgical therapy. ${ }^{3,4}$ Scaling and root planing is not predictable in eliminating pathogens completely from deep pockets, dentinal tubules, furcations, and other subgingival areas where access is poor. Use of systemic antibiotics which reach connective tissue site via serum, have shown to eliminate invading organisms. ${ }^{5}$ Metronidazole and tetracycline are the drugs most extensively documented by clinical trials and reports on their relative efficacy in supporting mechanical therapy of periodontitis. ${ }^{6}$ Clinical trials on subgingival pathogens found in chronic periodontitis patients have shown that Prevotella intermedia, Prevotella nigrescens, and A. actinomycetemcomitans are often resistant to doxycycline, amoxicillin, or metronidazole. ${ }^{3,6}$

Ofloxacin is a second-generation fluoroquinolone, broadspectrum antibiotic that is active against both gram-positive and
${ }^{1-3}$ Department of Periodontics, Sri Hasanamba Dental College and Hospital, Hassan, Karnataka, India

Corresponding Author: Harsha M Babu, Department of Periodontics, Sri Hasanamba Dental College and Hospital, Hassan, Karnataka, India, Phone: +91 9845735007, e-mail: harshamb@yahoo.com

How to cite this article: Chermmal AK, Babu HM, Vivekananda MR. Evaluation of Ofloxacin and Ornidazole as an Adjunct to Scaling and Root Planing in the Treatment of Generalized Chronic Periodontitis: A Randomized Clinical Study. World J Dent 2021;12(6):485-491.

Source of support: Nil

Conflict of interest: None

gram-negative bacteria. It functions by inhibiting two bacterial type II topoisomerases, DNA gyrase and topoisomerase IV. Ofloxacin is more completely absorbed, achieves higher peak serum concentrations, and has a longer terminal elimination half-life, which results in a fivefold greater area under the curve than that of ciprofloxacin when similar doses are orally administered. ${ }^{7}$

Ornidazole is a nitroimidazole derivative, acts by inhibiting DNA synthesis. The nitro group reduced to anion radical targets DNA, which oxidizes, leading to strand breakage and cell death of microorganisms. Ornidazole showed an increased half-life of elimination from plasma (14.4 vs 8.4 hours for metronidazole), therefore, require less frequent intake, which is twice daily. ${ }^{8}$ 
Since chronic periodontitis is of polymicrobial origin, combination therapy may yield better resolution as compared to single antibiotic therapy. The advantages of combination therapy are: (i) It may help to broaden the anti-microbial range of therapeutic regimens beyond that attained by any single antibiotic. (ii) It could prevent the development of bacterial resistance to a single antibiotic, and (iii) It lowers the dose of individual antibiotics by bringing about the synergy between the drugs against targeted organisms. ${ }^{8}$ To the best of our knowledge, studies reporting the utility of this ofloxacin-ornidazole combination in the treatment of generalized chronic periodontitis are scarce in the literature. Hence, this study was aimed to evaluate the efficacy of the ofloxacinornidazole combination as an adjunct to scaling and root planing in the treatment of generalized chronic periodontitis.

\section{Materials and Methods}

This randomized controlled clinical study was conducted on individuals reporting to the Department of Periodontics of Sri Hasanamba Dental College and Hospital, Hassan, Karnataka. Fifty patients between 25 years and 55 years of age with generalized chronic periodontitis reporting to the Department of Periodontics of Sri Hasanamba Dental College and Hospital, Hassan, Karnataka were included in the study. A randomized controlled design was used. Simple randomization of the patients (1:1 allocation ratio) to the SRP + ofloxacin-ornidazole combination therapy or SRP alone group was carried out. The study was conducted from September 2019 through November 2020. The Institutional Ethical Committee (Reg. No: ECR/963/Inst/KA/2017) approved the study (Ref. No: $\mathrm{SHDCH} / 2018-19 / 2487)$. The trial protocol was registered on Clinical Trials Registry-India (CTRI/2021/02/031487). Patients were screened for clinical signs consistent with the diagnosis of generalized chronic periodontitis and for eligibility to participate in the clinical trial. Patients satisfying all of the following inclusion criteria were enrolled in the clinical trial: (1) willingness to comply with the study protocol; (2) systemically healthy individuals between the age group of 25 and 55 range; (3) study participants who were diagnosed with periodontal pocket depth $\geq 5 \mathrm{~mm}$. Patients meeting any of the following exclusion criteria were not enrolled in the clinical trial: (1) study participants suffering from any known systemic diseases; (2) study participants who were smokers; (3) pregnant and lactating individuals; (4) study participants who had received any periodontal therapy/antibiotic therapy in the last 6 months; (5) unable or not willing to comply with the study protocol.

Of the total 45 individuals examined for eligibility, 40 individuals were selected for the study. The sample size was calculated using $\mathrm{G}$ * Power software (Heinrich-Heine-University Dusseldorf) with an a-error probability of $0.05 \%$ and $80 \%$ of statistical power. Forty individuals meeting the inclusion criteria were randomly assigned to one of the two following groups with a toss of a coin, 20 in each group.

- Group I: Receiving scaling and root planing (SRP) along with ofloxacin $(200 \mathrm{mg})+$ ornidazole $(500 \mathrm{mg})$ combination therapy for 5 days.

- Group II: Receiving scaling and root planing alone.

\section{Measurement of Study Variables}

The protocol comprised three visits for each patient with generalized chronic periodontitis, distributed over a period of 30 days. All the participants were explained about the purpose of the study and written informed consent was obtained before undertaking the study. Patients underwent full periodontal assessment at baseline, at 14 days, and at 1 month by the same examiner. Clinical examination included: (i) gingival index (GI) ${ }^{9}$ (ii) sulcular bleeding index (SBI), ${ }_{10}$ (iii) the probing pocket depth (PPD), ${ }^{11}$ at six sites per tooth with William's periodontal probe. Probing depth was measured in millimeters from the gingival margin to the depth of the periodontal pocket. At the baseline visit, full mouth scaling and root planing were performed for both groups and oral hygiene instructions are given.

\section{Statistical Analysis}

Statistical analysis was done using SPSS software version 20 and Microsoft Excel version 2007. Descriptive statistics will be done by measuring mean, standard deviation, and proportion. Inferential statistics will be done by unpaired $t$-test and chi-square test. For all statistical tests, $p$ value $<0.05$ was considered statistically significant.

\section{Results}

This randomized clinical trial was conducted to study the efficacy of systemically administered ofloxacin-ornidazole combination therapy as an adjunct to scaling and root planing on clinical parameters and to compare and evaluate the efficacy of systemically administered ofloxacin-ornidazole combination therapy with scaling and root planing alone.

Table 1 shows the frequency of gender distribution in the present study. Group I consisted of 9 males and 11 females. Group II consisted of 10 males and 10 females. There was no statistically significant difference in the proportion of male and female study participants between groups I and II.

Table 2 shows the frequency of age distribution in the present study. The mean age in group I was $41.6 \pm 7.214$ and in group II mean age was $42.15 \pm 9.314$. There was no statistically significant difference in mean age between group I and group II.

Table 3 and Figure 1 show the intergroup comparison of the gingival indices at baseline, 14th day, and 1 month among both the groups. The mean GI score at baseline was $1.9610 \pm 0.416$ for group I and $2.0495 \pm 0.329$ for group II. There was no statistically significant difference in the mean GI score between the groups at baseline. The mean Gl score on the 14th day was $1.0475 \pm 0.351$ for group I and $1.4375 \pm 0.307$ for group II. There was a statistically significant difference in the mean $\mathrm{Gl}$ score between the groups on the 14th day $(p=0.001)$. The mean $\mathrm{Gl}$ score at 1 month was $0.7060 \pm 0.368$ for group I and $1.2300 \pm 0.431$ for group II. There was a statistically significant difference in the mean $\mathrm{Gl}$ score between the groups at 1 month $(p<0.001)$.

Table 4 and Figure 2 show the intergroup comparison of the sulcular bleeding indices at baseline, 14th day, and 1 month among both the groups. The mean bleeding index score at baseline was $1.9065 \pm 0.505$ for group I and $1.7360 \pm 0.268$ for group II. There was no statistically significant difference in the mean GI score between the groups at baseline. The mean bleeding index score on the 14th day was $0.7875 \pm 0.365$ for group I and $1.1649 \pm 0.233$ for group II. There was a statistically significant difference in the mean bleeding index score between the groups on the 14th day $(p<0.001)$. The mean bleeding score at 1 month was $0.4940 \pm 0.321$ for group I and $0.9720 \pm 0.252$ for group II. There was a statistically significant difference in the mean bleeding index score between the groups at 1 month $(p<0.001)$. 
Table 1: Frequency distribution for gender between groups

\begin{tabular}{|c|c|c|c|c|c|}
\hline \multicolumn{6}{|c|}{ Chi-square test } \\
\hline & & & \multicolumn{2}{|c|}{ Group } & \multirow[b]{2}{*}{$p$} \\
\hline & & & Group I & Group II & \\
\hline \multirow[t]{6}{*}{ Sex } & Female & Count & 11 & 10 & 0.752 \\
\hline & & $\%$ within group & 55.0 & 50.0 & \\
\hline & Male & Count & 9 & 10 & \\
\hline & & $\%$ within group & 45.0 & 50.0 & \\
\hline & \multirow[t]{2}{*}{ Total } & Count & 20 & 20 & \\
\hline & & $\%$ within group & 100.0 & 100.0 & \\
\hline
\end{tabular}

Group I, ofloxacin-ornidazole combination therapy along with scaling and root planing; group II, scaling and root planing; $p$ probability. $p<0.05$ is considered to be statistically significant

Table 2: Frequency distribution for age between groups

\begin{tabular}{llllll}
\hline \multicolumn{5}{c}{ Unpaired t-test } \\
\hline Age & Group & $N$ & Mean & SD & $p$ \\
& Group I & 20 & 41.60 & 7.214 & 0.836 \\
& Group II & 20 & 42.15 & 9.314 & \\
\hline
\end{tabular}

Group I, ofloxacin-ornidazole combination therapy along with scaling and root planing; group II, scaling and root planing; $N$, number of subjects; SD, standard deviation, $p$, probability. $p<0.05$ is considered to be statistically significant

Table 3: Intergroup comparison of gingival indices at baseline, 14th day, and 1 month

\begin{tabular}{|c|c|c|c|c|c|}
\hline \multicolumn{6}{|c|}{ Unpaired t-test } \\
\hline & Group & $N$ & Mean & $S D$ & $p$ \\
\hline \multirow[t]{2}{*}{ Gl BL } & Group I & 20 & 1.96 & 0.416 & 0.461 \\
\hline & Group II & 20 & 2.04 & 0.329 & \\
\hline \multirow[t]{2}{*}{ Gl 14} & Group I & 20 & 1.04 & 0.351 & $0.001^{*}$ \\
\hline & Group II & 20 & 1.43 & 0.307 & \\
\hline \multirow[t]{2}{*}{ Gl 30} & Group I & 20 & 0.70 & 0.368 & $<0.001^{*}$ \\
\hline & Group II & 20 & 1.23 & 0.431 & \\
\hline
\end{tabular}

Group I, ofloxacin-ornidazole combination therapy along with scaling and root planing; group II, scaling and root planing; $N$, number of subjects, SD, standard deviation; $p$, probability. ${ }^{*} p<0.05$ is considered to be statistically significant

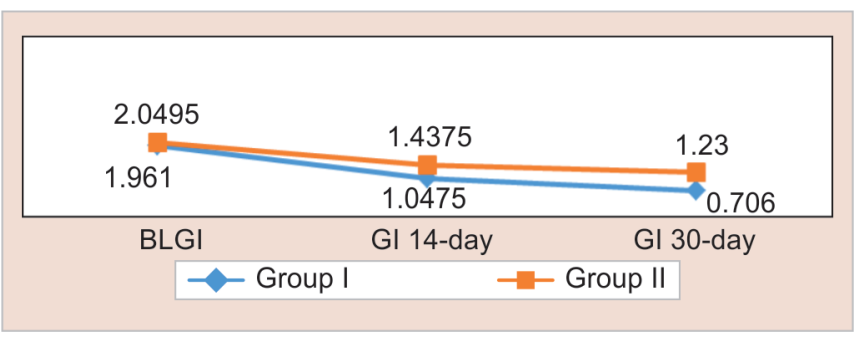

Fig. 1: Intergroup comparison of gingival indices at baseline, 14th day, and 1 month. Gl-gingival index, BLGI-Baseline gingival index

Table 5 and Figure 3 show the intergroup comparison of the PPD at baseline, 14th day, and 1 month among both the groups. The mean PPD score at baseline was $3.8475 \pm 0.448$ for group I and $3.7745 \pm 0.512$ for group II. There was no statistically significant difference in mean PPD score between the groups at baseline. The mean PPD score on the 14th day was $2.8005 \pm 0.446$ for group I and $3.2790 \pm 0.371$ for group II. There was a statistically significant difference in the mean PPD score between the groups on the 14th day $(p=0.001)$. The mean PPD at 1 month was $2.2625 \pm 0.311$ for group I and $2.9220 \pm 0.359$ for group II. There was a statistically significant difference in mean PPD score between the groups at 1 month $(p<0.001)$.

Table 6 shows the intergroup comparison of improvement in gingival indices on the 14th day and 1 month among both the groups. The mean GI improvement score on the 14th day was $46.5459 \pm 13.927$ for group I and $29.7531 \pm 10.766$ for group II. It shows there is a statistically significant difference in mean Gl improvement score between the groups on the 14th day $(p<0.001)$. The mean $\mathrm{Gl}$ improvement score at 1 month was $64.8269 \pm 14.673$ for group I and $40.6337 \pm 17.719$ for group II. It shows there is a statistically significant difference in mean Gl improvement score between the groups at 1 month $(p<0.001)$.

Table 7 shows the intergroup comparison of improvement in sulcular bleeding indices on the 14th day and 1 month among both the groups. The mean bleeding index improvement score on the 14 th day was $58.4950 \pm 13.938$ for group I and $32.0214 \pm 14.074$ for group II. It shows there is a statistically significant difference 
Table 4: Intergroup comparison of bleeding indices at baseline, 14th day, and 1 month

\begin{tabular}{llllll}
\hline \multicolumn{5}{c}{ Unpaired t-test } \\
\hline SBI BL & Group & $N$ & Mean & SD & 0.190 \\
& Group I & 20 & 1.90 & 0.505 & 0.268 \\
SBI 14 & Group II & 20 & 1.73 & 0.365 & $<0.001^{*}$ \\
& Group I & 20 & 0.78 & 0.233 & $<0.001^{*}$ \\
SBI 30 & Group II & 20 & 1.16 & 0.321 & 0.252 \\
& Group I & 20 & 0.49 & 0.97 & \\
\hline
\end{tabular}

Group I, ofloxacin-ornidazole combination therapy along with scaling and root planing; group II, scaling and root planing; $N$, number of subjects; SD, standard deviation; $p$, probability. ${ }^{*} p<0.05$ is considered to be statistically significant

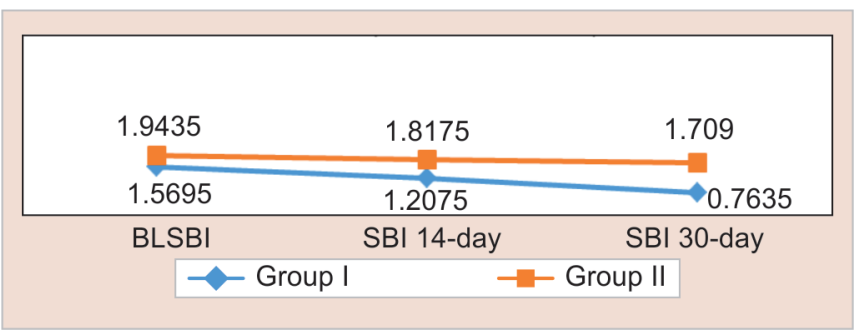

Fig. 2: Intergroup comparison of sulcular bleeding indices at baseline, 14th day, and 1 month. SBI-Sulcus bleeding Index, BLSBI-Baseline sulcus bleeding index

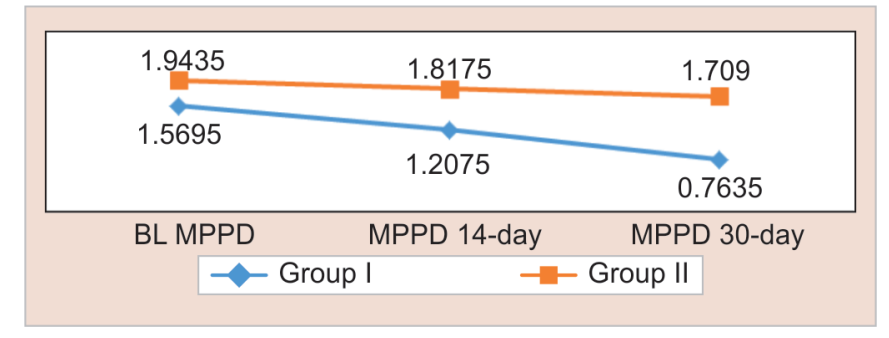

Fig. 3: Intergroup comparison of probing pocket depth at baseline, 14th day, and 1 month. PPD-mean probing pocket depth, BL MPPD-baseline mean probing pocket depth

Table 5: Intergroup comparison of probing pocket depth at baseline, 14th day, and 1 month

\begin{tabular}{llllll}
\hline \multicolumn{5}{c}{ Unpaired t-test } \\
\hline MPPD BL & Group & $N$ & Mean & SD & 0.634 \\
& Group I & 20 & 3.84 & 0.448 & 0.512 \\
MPPD 14 & Group II & 20 & 3.77 & 0.446 & $0.001^{*}$ \\
& Group I & 20 & 2.80 & 0.371 & $<0.001^{*}$ \\
MPPD 30 & Group II & 20 & 3.27 & 0.311 & 0.359 \\
& Group I & 20 & 2.26 & 2.92 & \\
\hline
\end{tabular}

Group I, ofloxacin-ornidazole combination therapy along with scaling and root planing; group II, scaling and root planing; $N$, number of subjects; SD, standard deviation; $p$, probability. ${ }^{*} p<0.05$ is considered to be statistically significant

Table 6: Intergroup comparison of improvement in gingival indices at 14th day and 1 month

\begin{tabular}{llllll}
\hline \multicolumn{7}{c}{ Unpaired t-test } \\
\hline GI IMP 14 & Group & $N$ & Mean & SD & PD \\
& Group I & 20 & 46.54 & 13.927 & $<0.001^{*}$ \\
GI IMP 30 & Group II & 20 & 29.75 & 10.766 & $<0.001^{*}$ \\
& Group I & 20 & 64.82 & 14.673 & 17.719 \\
\hline
\end{tabular}

Group I, ofloxacin-ornidazole combination therapy along with scaling and root planing; group II: scaling and root planing; $N$, number of subjects; SD, standard deviation; $p$, probability. ${ }^{*} p<0.05$ is considered to be statistically significant

in the mean bleeding index improvement score between the groups on the 14th day ( $p<0.001)$. The mean bleeding index improvement score at 1 month was $75.0530 \pm 11.003$ for group I and $42.5086 \pm 17.428$ for group II. It shows there is a statistically significant difference in mean bleeding index improvement score between the groups at 1 month $(p<0.001)$.

Table 8 shows the intergroup comparison of improvement in PPD on the 14th day and 1 month among both the groups. The mean PPD improvement score on the 14th day was $27.2930 \pm$ 7.441 for group I and $12.6973 \pm 5.941$ for group II. It shows there is a statistically significant difference in mean PPD improvement score between the groups on the 14 th day $(p<0.001)$. The mean PPD improvement score at 1 month was $40.9764 \pm 7.025$ for group I and $22.0287 \pm 8.211$ for group II. It shows there is a statistically significant difference in mean PPD improvement score between the groups at 1 month $(p<0.001)$. 
Evaluation of Ofloxacin and Ornidazole as an Adjunct to Scaling and Root Planing

Table 7: Intergroup comparison of improvement in sulcular bleeding indices at 14th day and 1 month

\begin{tabular}{llllll}
\hline \multicolumn{7}{c}{ Unpaired t-test } \\
\hline \multirow{2}{*}{ SBI IMP 14 } & Group & $N$ & Mean & SD & P \\
& Group I & 20 & 58.49 & 13.938 & $<0.001^{*}$ \\
SBI IMP 30 & Group II & 20 & 32.02 & 14.074 & $<0.001^{*}$ \\
& Group I & 20 & 75.05 & 11.003 & 17.428 \\
\hline
\end{tabular}

Group I, ofloxacin-ornidazole combination therapy along with scaling and root planing; group II: scaling and root planing; $N$, number of subjects; SD, standard deviation; $p$, probability. ${ }^{*} p<0.05$ is considered to be statistically significant

Table 8: Intergroup comparison of improvement in probing pocket depth at the 14th day and 1 month

\begin{tabular}{llllll}
\hline \multicolumn{1}{c}{} & \multicolumn{5}{c}{ Unpaired t-test } \\
\hline MPPD IMP 14 & Group & $N$ & Mean & SD & $<0.001^{*}$ \\
& Group I & 20 & 27.29 & 7.441 & $<.941$ \\
MPPD IMP 30 & Group II & 20 & 12.69 & 7.025 & $<0.001^{*}$ \\
& Group I & 20 & 40.97 & 8.211 & \\
\hline
\end{tabular}

Group I, ofloxacin-ornidazole combination therapy along with scaling and root planing; group II: scaling and root planing; $N$, number of subjects, SD, standard deviation; $p$, probability. ${ }^{*} p<0.05$ is considered to be statistically significant

\section{Discussion}

Periodontitis is a chronic inflammatory disease that affects the supporting structures of teeth, resulting in tooth loss. Conventional periodontal therapy includes both surgical and non-surgical approaches that involve instrumentation of the inflamed dentogingival complex. ${ }^{12}$ Scaling and root planing is an important component of nonsurgical periodontal therapy in the treatment of generalized chronic periodontitis. Factors such as furcation involvement, tooth type, surface, the experience of the operator, and the tissue invasive nature of the pathogens, limit its effectiveness. ${ }^{13}$ Several studies that assessed the adequacy of a combination of SRP and systemic antibiotics in therapy of chronic periodontitis have concluded that combining systemic antibiotics with SRP is more advantageous than SRP alone. . $^{14,15}$

However, the development of antibiotic resistance has increased in recent times with the use of commonly used antibiotics. An in vitro study regarding antibiotic resistance reported $74.2 \%$ of the patients with $\mathrm{CP}$ revealed subgingival periodontal pathogens resistant to at least one of the test antibiotics. At least one test organism, P. intermedia, Streptococcus constellatus, or A. actinomycetemcomitans were protected in vitro to doxycycline, amoxicillin, metronidazole, or clindamycin, in patients with chronic periodontitis. Fifteen percent of patients held subgingival periodontal microorganisms impervious to both amoxicillin and metronidazole, which were for the most part either S. constellatus or ciprofloxacin-susceptible strains of gram-negative enteric rods/Pseudomonas. ${ }^{16,17}$

Periodontal infections are of polymicrobial origin involving a variety of gram-positive and gram-negative, microaerophilic, anaerobic, and aerobic bacteria, hence no single antibiotic is effective against all putative pathogens. ${ }^{18}$ Thus, the use of more than one antibiotic, serially or in combination becomes necessary. ${ }^{19}$ The current study attempted to evaluate the efficacy of one such combination therapy which was composed of ofloxacin-ornidazole as an adjunct to scaling and root planing.
Ofloxacin belongs to the fluoroquinolone family which is effective against the Pasteurella family to which $A$. actinomycetemcomitans belongs. ${ }^{20}$ Miyake et al. examined the antibacterial activity of ofloxacin against periodontal diseaseassociated bacteria in an in vitro study. Ofloxacin showed marked antibacterial activity against periodontal disease-associated bacteria. MICs against Bacteroides asaccharolyticus, B. gingivalis, all strains of $A$. actinomycetemcomitans, and Capnocytophaga sputigena were lower than $0.006 \mu \mathrm{g} / \mathrm{mL}$. They also concluded that the antibacterial activity of ofloxacin might not disturb the normal bacterial flora. ${ }^{21}$ Higashi et al. showed that following oral administration of $200 \mathrm{mg}$ of ofloxacin, its concentration in GCF increased rapidly, being 2 or 3 times higher than that in saliva. ${ }^{22}$ The adverse effects of ofloxacin reported were similar to or significantly less frequent than those reported with other antibiotics. ${ }^{23}$ Schwartz and Jeunet reported that the half-life of ornidazole in human plasma is found to be 1.7 times longer than that of metronidazole requiring less frequency of intake. ${ }^{24}$ Ellie and Vera have reported that ornidazole requires the least minimum inhibitory concentration to repress the growth of periodontal pathogens when contrasted with that of metronidazole. ${ }^{25}$ Mombelli et al. conducted a study to evaluate the use of ornidazole as an adjunct to root planing in the therapy of patients suffering from recurrent periodontal disease. A study found out that ornidazole has an additional, beneficial clinical and microbiological effect when used as an adjunct to mechanical re-treatment of recurrent periodontitis. ${ }^{6}$ Pradeep et al. conducted a randomized clinical trial of 6 months duration to evaluate the effect of systemic ornidazole on total anaerobic microbial counts of subjects with moderate to advanced chronic periodontitis. Their study showed that the systemic use of ornidazole, when used in conjunction with initial periodontal treatment consisting of SRP in adult subjects with periodontitis, achieves significantly better clinical results than initial periodontal treatment alone. ${ }^{26}$

In our study, group I patients underwent scaling and root planing and received ofloxacin-ornidazole combination therapy 
Flowchart 1: Consort chart

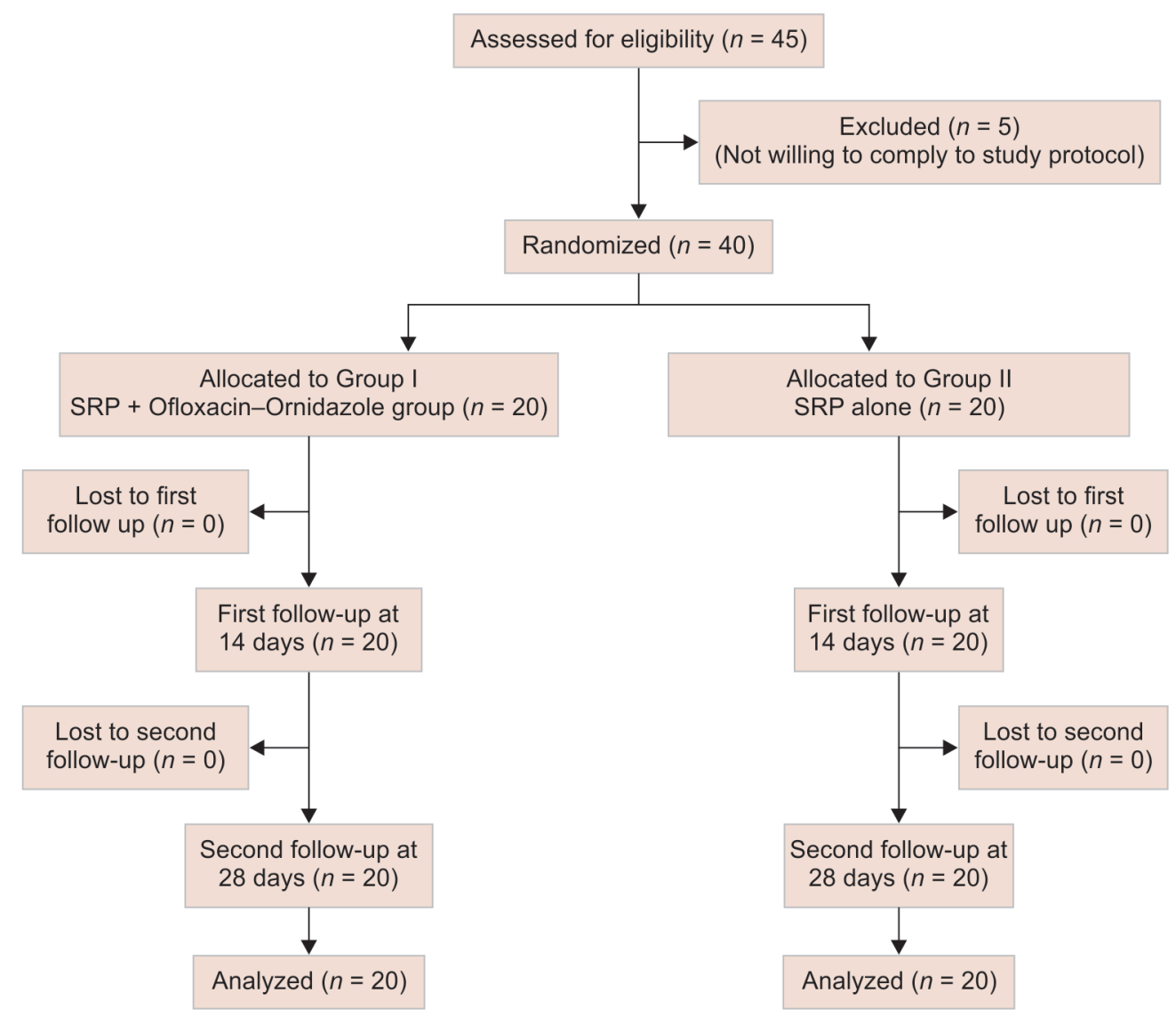

for 5 days as an adjunct, and group II patients underwent scaling and root planing alone. On analyzing the results obtained, we found that from baseline to 14th day and to 1 month were comparable on intergroup analysis which showed a statistically significant difference on all the three clinical parameters. An intergroup analysis of mean improvement scores of the Gl, bleeding index, and PPD from baseline to 14th day and 1 month showed a higher improvement score in group I than group II and it was statistically significant. No side effects were reported.

The results of our study were similar to the findings of Boid et al. ${ }^{2}$ who compared the efficacy of ofloxacin-ornidazole combination therapy and azithromycin as an adjunct to scaling and root planing in periodontitis patients. The results of our study are in concurrence with the findings of Prathiba and Mahesh Kumar, who conducted a study to compare the efficacy of a combination of amoxicillin + metronidazole and ofloxacin + ornidazole in the treatment of a dentoalveolar abscess. They concluded that a combination of ofloxacin and ornidazole therapy is as efficient as amoxicillin and metronidazole therapy in the treatment of a dentoalveolar abscess. ${ }^{27}$ Eisen et al. conducted a study to determine the relationship between prescribed daily dose frequency and patient medication compliance. They concluded that probably the single most important action that healthcare providers can take to improve compliance is to select medications that permit the lowest daily prescribed dose frequency. This finding supports our present study's conclusion of favorable patient compliance of ofloxacin-ornidazole combination. ${ }^{28}$

\section{CONCLUSION}

The main finding of this study showed improved periodontal status and a significant improvement in clinical parameters after nonsurgical periodontal treatment when ofloxacin-ornidazole combination therapy was administered as an adjunct. Based on the findings of our study, we can suggest the use of the ofloxacinornidazole combination as an adjunct in the treatment of generalized chronic periodontitis. Further studies should be carried out with larger samples, varying time period of trial to establish its efficacy in the treatment of chronic periodontitis and open new doors in the field of research in periodontitis (Flowchart 1).

\section{References}

1. Socransky SS, Haffajee AD. Periodontal microbial ecology. Periodontol 2000 2005;38(1):135-187. DOI: 10.1111/j.1600-0757.2005.00107.x.

2. Boid A, Muglikar S, Hegde R. A comparative evaluation of systemic azithromycin and ornidazole - ofloxacin combination as an adjunct to scaling and root planing in the treatment of chronic generalised periodontitis. Dentistry 2015;5(07):309. DOI: 10.4172/21611122.1000309 .

3. Walters J, Pin-Chuang L. Should antibiotics be prescribed to treat chronic periodontitis? Dent Clin N Am 2015;59(4):919-933. DOI: 10.1016/j.cden.2015.06.011.

4. Bragd L, Dahlen G, Wikstrom M, et al. The capability of Actinobacillus actinomycetem comitans, Bacteroides gingivalis and Bacteroides intermedius to indicate progressive periodontitis; a retrospective study. J Clin Periodontol 1987;14(2):95-99. DOI: 10.1111/j.1600051x.1987.tb00949.x. 
5. Zandbergen D, Slot $D$, Cobb C, et al. The clinical effect of scaling and root planing and the concomitant administration of systemic amoxicillin and metronidazole: a systematic review. J Periodontol 2013;84(3):332-351. DOI: 10.1902/jop.2012.120040.

6. Mombelli A, Gusbertil FA, Lang NP. Treatment of recurrent periodontal disease by root planing and ornidazole. J Clin Periodontol 1989;16(1):38-45. DOI: 10.1111/j.1600-051x.1989.tb01610.x.

7. Wolfson JS, Hooper DC. Comparative pharmacokinetics of ofloxacin and ciprofloxacin. Am J Med 1989;87(6C):315-320.

8. Patil V, Mali R, Mali A. Systemic anti-microbial agents used in periodontal therapy. J Indian Soc Periodontol 2013;17(2):162-168. DOI: 10.4103/0972-124X.113063.

9. Loe $\mathrm{H}$, Silness J. Periodontal disease in pregnancy. I. Prevalence and severity. Acta Odontol Scand 1963;21(6):533-551. DOI: 10.3109/00016356309011240.

10. Carter HG, Barnes GP. The gingival bleeding index. J Clin Periodontol 1974;45(11):801-805. DOI: 10.1902/jop.1974.45.11.801.

11. Singh S, Vandana KL. Periodontal measurements: a dilemma. Indian J Multidiscip Dent 2018;8:17-20.

12. Badersten A, Nilve'us R, Egelberg J. Effect of nonsurgical periodontal therapy. I. Moderately advanced periodontitis. J Clin Periodontol 1981;8(1):57-72. DOI: 10.1111/j.1600-051x.1981.tb02024.x.

13. Brayer WK, Mellonig JT, Dunlap RM. Scaling and root planing effectiveness: the effect of root surface access and operator experience. J Periodontol 1989;60(1):67-72. DOI: 10.1902/ jop.1989.60.1.67.

14. Herrera D, Sanz M, Jepsen S. A systematic review on the effect of systemic antimicrobials as an adjunct to scaling and root planing in periodontitis patients. J Clin Periodontol 2002;29:136-159. DOI: 10.1034/j.1600-051x.29.s3.8.x.

15. Haffajee AD, Socransky SS, Gunsolley JC. Systemic anti-infective periodontal therapy: a systematic review. Ann Periodontol 2003;8(1):115-181. DOI: 10.1902/annals.2003.8.1.115.

16. Rams TE, Degener JE, van Winkelhoff AJ. Antibiotic resistance in human chronic periodontitis microbiota. J Periodontol 2014;85(1):160-169. DOI: 10.1902/jop.2013.130142.

17. Dupin C, Tamanai-Shacoori Z, Ehrmann E. Oral gram-negative anaerobic bacilli as a reservoir of b-lactam resistance genes facilitating infections with multiresistant bacteria. Int J Antimicrob Agents 2015;45(2):99-105. DOI: 10.1016/j.ijantimicag.2014. 10.003 .

18. Walker CB, Gordon JM, Magnusson I, et al. A role for antibiotics in the treatment of refractory periodontitis. J Periodontol 1993;64(8s):772781. DOI: 10.1902/jop.1993.64.8s.772.

19. Rams TE, Slots J. Antibiotics in periodontal therapy: an update. Compendium 1992;13(12):1130-1134.

20. Tanner A, Maiden MF, Paster BJ. The impact of $16 \mathrm{~S}$ ribosomal RNA based phylogeny on the taxonomy of oral bacteria. Periodontol 2000 1994;5(1):26-51. DOI: 10.1111/j.1600-0757.1994.tb00017.x.

21. Miyake $\mathrm{Y}$, Onoe $\mathrm{T}$, Sagawa $\mathrm{H}$, et al. In vitro antibacterial activity of ofloxacin against periodontal disease-associated bacteria. Periodont Res 1988;23(3):222-223. DOI: 10.1111/j.1600-0765.1988. tb01361.x.

22. Higashi K, Seike M, Mitani Y, et al. Concentration of ofloxacin in human gingival crevicular fluid after oral administration of tarivid. J Periodont Res 1989;24(6):409-411. DOI: 10.1111/j.1600-0765.1989. tb00890.x.

23. Hooper DC, Wolfson JS. The fluoroquinolones; pharmacology, clinical uses, and toxicities in humans. Antimicrob Agents Chemother 1985;28(5):716-721. DOI: 10.1128/AAC.28.5.716.

24. Schwartz DE, Jeunet F. Comparative pharmacokinetic studies of ornidazole and metronidazole in man. Chemotherapy 1976;22(1):1929. DOI: $10.1159 / 000221906$.

25. Ellie JC, Vera LS. Comparative susceptibility of anaerobic bacteria to metronidazole and ornidazole. Anti Microbial Agents Chemother 1978;14(4):609-613. DOI: 10.1128/AAC.14.4.609.

26. Pradeep AR, Kalra N, Priyanka N, et al. Microbiological outcomes of systemic ornidazole use in chronic periodontitis. Part II. J Int Acad Periodontol 2012;14(2):50-54.

27. Prathiba S, Mahesh Kumar PA. Comparative study of the combination of drugs -amoxicillin with metronidazole and ofloxacin with ornidazole in the treatment of dentoalveolar abscess. Oral Health Case Rep 2018;4:143-148.

28. Eisen SA, Miller DK, Woodward RS, et al. The effect of prescribed daily dose frequency on patient medication compliance. Archi Int Med 1990;150(9):1881-1884. DOI: 10.1001/archinte.1990.00390200073014. 